\title{
Strategic Planning towards Automation of Fiber To The Home (FTTH) Considering Optic Access Network (OAN) Model
}

\author{
Abid Naeem ${ }^{1}$, Shahryar Shafique ${ }^{2}$ \\ Department of Electrical Engineering \\ Iqra National University, Peshawar, Pakistan \\ Zahid Wadud ${ }^{3}$ \\ Department of Computer System Engineering \\ University of Engineering and Technology \\ Peshawar, Pakistan
}

\author{
Sheeraz Ahmed ${ }^{4}$ \\ Department of Computer Science \\ Iqra National University, Peshawar, Pakistan
}

\author{
Nadeem Safwan ${ }^{5}$ \\ Department of Management Sciences \\ Iqra National University \\ Peshawar, Pakistan
}

\author{
Zeeshan Najam ${ }^{6}$ \\ Department of Electrical Engineering \\ Ultimate Consultancy, Peshawar, Pakistan
}

\begin{abstract}
With the intention to meet the increasing demand of future higher bandwidth applications, fiber based Gigabit Passive Optical Network (GPON) access is considered best resolution to deliver triple play services (voice, data, video). Hence, it becomes obligatory to migrate from traditional copperbased network to fiber-based. Due to rapid technological evolution, tough competition and budget limitation the service providers are struggling to provide a cost effective solution to minimize their operational cost with extra ordinary customer satisfaction. One of the factors that increase the cost of overall Fiber To The Home (FTTH) network is the unplanned deployment resulting in utilization of extra components and resources. Hence, it is imperative to determine a suitable technique, which helps to reduce planning process, required time and deployment cost through optimization. Automation based planning is one of the possible ways to automate the network design at probable lowest cost. In this research, a planning technique for migration from copper to fiber access network with a manageable and optimized Passive Optic Network (PON FTTx) infrastructure is presented identifying a cost-effective strategy for developing countries.
\end{abstract}

Keywords-Fiber To The Home; Passive Optical Networks; GPON; triple play; cost effective; customer satisfaction

\section{INTRODUCTION}

In order to provides a triple-play (voice, data, video) services by high speed collaborating apps, like games that runs online, several telecommunication organizations are considering Fiber based accessed networks as the key resolution. There are two different ways of delivering fiber networks to customer premises, namely Point-to-Point (P2P) and Point to Multi-Point (P2MP). On one hand, P2P [1] fiber networks use a specific fiber constituent to link specific customer sites all the way to exchange. It allows very high bandwidth services (compared with P2MP) to be delivered to businesses or high rise buildings even over a long distance. However, when the number of P2P connections is very high, the installation and maintenance costs can be prohibitively expensive.

On the other hand, the P2MP network based on GPON technology can provide an attractive solution to reduce the overall cost. With the P2MP GPONs, there are no electronic components between an exchange and customer premises. Only optical splitters are used to connect Optical Line Terminal (OLT) equipment at an exchange to a group of premises sharing the same feeder fiber. An Optical Network Unit (ONU) will then be used to convert the optical signal into an electronic signal at the customer's premises.

GPON has a downstream capacity of $2.488 \mathrm{~Gb} / \mathrm{s}$ and an upstream capacity of $1.244 \mathrm{Gbp} / \mathrm{s}$ that is shared among users. GPONs are generally considered to be a more cost effective way of delivering FTTH services with minimum number of fibers and electronics required.

According to the market research division of Light Reading [2], the number of households with fiber-optic network connections was expected to grow by more than $32 \%$ worldwide in 2009 and will continue to grow at rates close to $45 \%$ a year through 2021. The number of fiber-connected households will reach nearly 130 million globally by that time.

Though, to form a cost effective GPON/FTTHN needs consideration of different factors, such as, locations of splitters, cable assignment of customer sites to splitters and provision of spare capacity for future growth. In addition, all the planning restraints like extreme allowable splitters capability and the extreme distance in OLTs, ONUs, splitters satisfied must. 
To plan a GPON/FTTH network manually in a new area, an organizer is usually given a background plan that assisted by exchange. Whole region is subdivided in to small zones that are settled in multiple phases. With given rules of planning and sites of locations, organizer typically positions the optical Splitters (SPs) somewhere in the center of the planned area. Cable Distribution points (CD)s will then be positioned around the SP afterwards. Once the locations of SPs and CDs are specified, the planner will assign cables from each customer premise to a CD and from a CD to a SP based on the shortest distance.

This manual design process is very time consuming. Very often, due to the tight time schedule, when the design proposed by the planner satisfies all the distance and capacity constraints, he/she will submit the design without incorporating much cost optimization or considering the distribution of spare capacity.

\section{A. Components of GPON FTTH Network}

A Passive Optical Network (PON) is capable of having P2M (point to multipoint) network with passive components like optical splitter or coupler along the transmission section. It uses active components only at $\mathrm{CO}$ and at customer premises. It uses WDM to mix up video signals with the data and voice from OLT. Fig. 1 shows the basic FTTH Network. [3].

1) Optical Line Terminal (OLT): It is the most important part of the network, where the electrical signal from the service provider's equipment are converted into optical signals and given to the feeder network. The mode of transmission from ONT is broadcasting [4] from where it sends GEM frames through the GEM port with GEM port IDs It is capable of having Multi-service chassis for FTTx deployments, Supports a variety of service types, Non-blocking architecture with \& Routing within distributed architecture, scalability and line rate performance, Full electrical and optical redundancy Outstanding scalability and line rate performance, Real-time network traffic monitoring and analysis.V8240 GPON OLT is used. Specifications are given in Table I.

2) Optical Network Terminal (ONT): It is an active component used at customer premises which converts optical to electrical signals. ONU/ONT represents the ingle customer where they will get the triple play application. H640 series GPON ONT are used. It is capable of having carrier class VoIP telephony supporting both MGCP and SIP protocols, Flexible VLAN tagging support, QoS for traffic prioritization and bandwidth management, IGMP support for IPTV applications. Its specifications are given in Table II.

3) Splitter: Splitters are used to physically split the fiber to number of fibers; to couple same or different information's to $\mathrm{N}$ users. MxN planar splitters are used which is based on planar light wave circuit (PLC) technology and high precision alignment. MxN splitters can split or combine light from one or two fibers into $\mathrm{N}$ outgoing fibers uniformly over a wide spectral range with ultra-low insertion loss and low polarization dependent loss. With up to 64 output ports, these splitters are ideal for high density split applications like Fiber
To The Home (FTTH) networks, FTTx Deployments Optical CATV Networks, CWDM and DWDM Systems, Passive Optical Networks, Fiber Communication Systems Telecom, LANs. It has the features like Low Insertion Loss, Ultra broadband performance $(1260-1630 \mathrm{~nm})$, Low PDL and PMD, Stable towards thermal variations, Superior port to port uniformity. A splitter type is shown in Fig. 2.

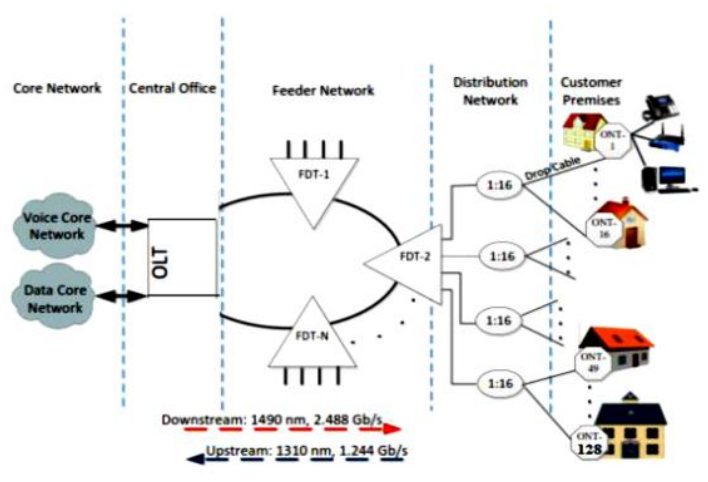

Fig. 1. FTTH Network [3].

TABLE. I. V8240 GPON OLT SPECIFICATIONS

\begin{tabular}{|l|l|}
\hline Flash Memory & $72 \mathrm{MB}$ \\
\hline SDRAM & $1 \mathrm{~GB}$ \\
\hline Dimensions (W x H x D) & $\begin{array}{l}17.1 \text { x } 12.2 \text { x } 11.2 \text { in }(434 \text { x } 310 \text { x } 285 \\
\text { mm }\end{array}$ \\
\hline Switching Capacity & $296 \mathrm{Gbps}$ \\
\hline Power Voltage AC type & $100-240 \mathrm{VAC}, 50 / 60 \mathrm{~Hz}$ \\
\hline DC type & $-48 / 60 \mathrm{VDC}$ \\
\hline Operating Temp & 32 to $122^{\circ} \mathrm{F}\left(0\right.$ to $\left.50^{\circ} \mathrm{C}\right)$ \\
\hline SIU (Subscriber Interface Unit) & 10 slots \\
\hline NIU (Network Interface Unit) & 2 slots \\
\hline SFU (Switching Fabric Unit) & 2 Slots \\
\hline
\end{tabular}

TABLE. II. H640 SERIES GPON ONT

\begin{tabular}{|c|c|}
\hline Service Interface & $\begin{array}{l}4 \text { 10/100Base-TX ports (RJ45) } \\
2 \text { POTS ports (RJ11) } \\
1 \text { RF video port (F-connector) }\end{array}$ \\
\hline Uplink Interface & 1 GPON port (SC/APC type) \\
\hline Operating Temp & 32 to $104^{\circ} \mathrm{F}\left(0\right.$ to $\left.40^{\circ} \mathrm{C}\right)$ \\
\hline Storage Temp & -4 to $140^{\circ} \mathrm{F}\left(-20\right.$ to $\left.60^{\circ} \mathrm{C}\right)$ \\
\hline Input & 100-240VAC \\
\hline Dimensions (W x H xD) & $\begin{array}{l}\text { - Excluding bracket: } 10.24 \times 2.05 \times 7.87 \text { in }(260 \times \\
52 \times 200 \mathrm{~mm}) \\
\text { - Including bracket, wall mounting: } 10.51 \times 2.60 \times \\
7.87 \text { in }(267 \times 66 \times 200 \mathrm{~mm}) \\
\text { - Excluding bracket, desktop mounting: } 10.24 \times \\
2.80 \times 7.87 \text { in }(260 \times 71 \times 200 \mathrm{~mm})\end{array}$ \\
\hline
\end{tabular}

Fig. 2. PLC Splitter with Ribbon Fiber. 


\section{Business Model of FtTH PlanNING}

\section{A. Business Model of FTTH Planning}

With the intention to meet the increasing demand of future higher bandwidth applications, the fiber based access is considered to be a best resolution to offer triple play services. It is therefore preferred with great need to migrate from traditional capper based network to fiber based access. A business Model of new FTTH network deployment is illustrated in Fig. 3 which consists of some dependent and independent variable.

In Pakistan the broadband growth in wireline is very slow which is very much obvious. In [7], the slow growth is due to some factors that need improvement; these factors include.

- Low literacy rate

- low (level of) consumer awareness

- No coverage of Broadband services

- Traffic reduction in broadband services low computer penetration

- Cost of service (tariff)

- History of market and national regulation

1) Business output: The output of new FTTH Deployment translates into different benefits [20].

- End User/Customer benefits

High bandwidth is the main selling product of FTTH network; it provides the highest available bandwidth in both directions (downstream and upstream). A FTTH user can download data over 10 times quicker than ADSL user. Transfer rate of different content over various types of networks are shown in Table III.

Speed of ADSL over Copper network is inversely proportional to distance from customer end to telephone exchange, while in FTTH network distance does not affect speed. In DSL network signal to noise ratio (SNR), interference and crosstalk during operation also reduces the throughput. Customer satisfaction ratio in FTTH network is above $85 \%$, higher customer satisfaction has a tendency to enhanced customer retention and reduce churn.

- Service provider benefits

$\checkmark \quad$ The lifespan of Fiber cable is very long as it is more than 30 years therefore FTTH is known as a "future-proof technology". Fiber cable made of simply plastic and glass, which reduce his lifespan extremely slowly. The fiber cable has almost unlimited capacity and expansion in bandwidth needs only changes to the hardware at the ends of the link.

$\checkmark$ Operational cost (OPEX) of FTTH networks is very low as compare to existing copper networks. It consumes 20 times less power than other. The operational and maintenance cost can be minimizing by automation control. Maintenance costs can also reduce because there is no active device in the field to maintain, and optical components have better reliability.

$\checkmark$ Customer satisfaction will reduce the churn value and increase the customer, which also reduce operational cost. To keep the existing customer is so easy as compare to enlist new one. To maintain an existing customer is so easier than to register a fresh customer.

- Community benefits

FTTH enable Communities can get a lot of benefits with a wider range of internet services. Few examples of possible benefits with FTTH networks are as under:

$>$ Financial boost with global competition.

$>$ Attraction for new businesses.

$>$ Provisioning of state of the art services in Education and health sector.

$>$ Improving overall quality of life in a community by increasing the opportunities for communication.

$>$ Controlling of Road traffic blocking/problem.

\section{Business Model at a glance}

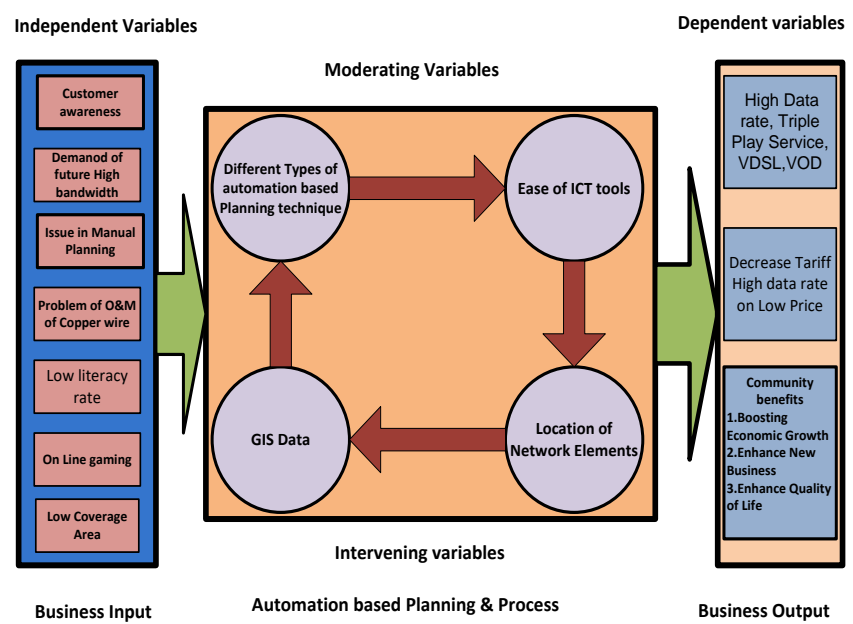

Fig. 3. Business Model of FTT H Optimization.

TABLE. III. DOWNLOAD/UPLOAD TRANSFER RATE [20]

\begin{tabular}{|l|l|l|l|l|l|l|}
\hline \multirow{2}{*}{ Data } & \multicolumn{2}{|l|}{ FTTH } & \multicolumn{2}{l|}{ CATV } & \multicolumn{2}{l|}{ DSL } \\
\cline { 2 - 7 } & Down & Up & Down & Up & Down & Up \\
\hline $\begin{array}{l}\text { Pictures up } \\
\text { to 1 GB }\end{array}$ & $\begin{array}{l}1 \mathrm{M} 23 \\
\mathrm{~S}\end{array}$ & $\begin{array}{l}1 \mathrm{M} \\
23 \mathrm{~S}\end{array}$ & $\begin{array}{l}2 \mathrm{M} 46 \\
\mathrm{~S}\end{array}$ & $\begin{array}{l}13 \mathrm{M} \\
52 \mathrm{~S}\end{array}$ & $19 \mathrm{M}$ & $\begin{array}{l}2 \mathrm{Hr} 32 \\
\mathrm{M}\end{array}$ \\
\hline $\begin{array}{l}\text { Standard } \\
\text { video Up } \\
\text { to 5 GB }\end{array}$ & $\begin{array}{l}6 \mathrm{M} 31 \\
\mathrm{~S}\end{array}$ & $\begin{array}{l}6 \mathrm{M} \\
31 \mathrm{~S}\end{array}$ & $\begin{array}{l}13 \mathrm{M} \\
2 \mathrm{~S}\end{array}$ & $\begin{array}{l}1 \mathrm{Hr} 5 \\
\mathrm{M}\end{array}$ & $\begin{array}{l}1 \mathrm{Hr} 29 \\
\mathrm{M}\end{array}$ & $\begin{array}{l}11 \mathrm{Hr} \\
29 \mathrm{M}\end{array}$ \\
\hline $\begin{array}{l}\text { HD } \\
\begin{array}{l}\text { Quality } \\
\text { Video up } \\
\text { to 25 GB }\end{array}\end{array}$ & $\begin{array}{l}24 \mathrm{M} \\
40 \mathrm{~S}\end{array}$ & $\begin{array}{l}24 \mathrm{M} \\
40 \mathrm{~S}\end{array}$ & $\begin{array}{l}1 \mathrm{H} 9 \\
\mathrm{M}\end{array}$ & $\begin{array}{l}5 \mathrm{Hr} \\
47 \mathrm{M}\end{array}$ & $\begin{array}{l}7 \mathrm{Hr} \\
\mathrm{M}\end{array}$ & $\ldots$ \\
\hline
\end{tabular}


2) Automation based planning and process: To grip complication of FTTHN, an automation scheme is settled by keeping in mind, common optimization framework, which shows in Fig. 4 with different Phases.

a) Input Phase: In this phase various sources can be used to retrieve the data like:

- Geo-graphical Information Systems (GIS)

- Manually formed files

GIS data contains the setup of access network. Usually it used a geographical database with a three-dimensional data structure. To get the quick recovery of information, it associates a wide range of geographic items with a rich set of attributes.

Manually created files are the second source of input data phase. A Map of an exchange or area is typically used by planner. The area further divided into different regions. The planners usually choose a central point of the region for installation of Main Distribution Box (MDB) which consists of various splitters. Moreover, they allocate cables from end user to splitters through distribution Cabinet (DC).

b) Input Analyzer Phase: As the data gathered by different sources are in different format such as DXF, Esri Shape, so this stage is utilized to filter the required data from Input source. The data is first transformed into some matrices which consist of required information to perform optimization procedures efficiently.

c) Business Logic Phase: The comprehensive cost model with engineering rules summarizes in this stage. To reduce the cost of network design problems the engineering rules used for constrains. Different costs of network design like HR cost, ducting costs, cabling and network equipment cost includes in this cost model.

Input Phase

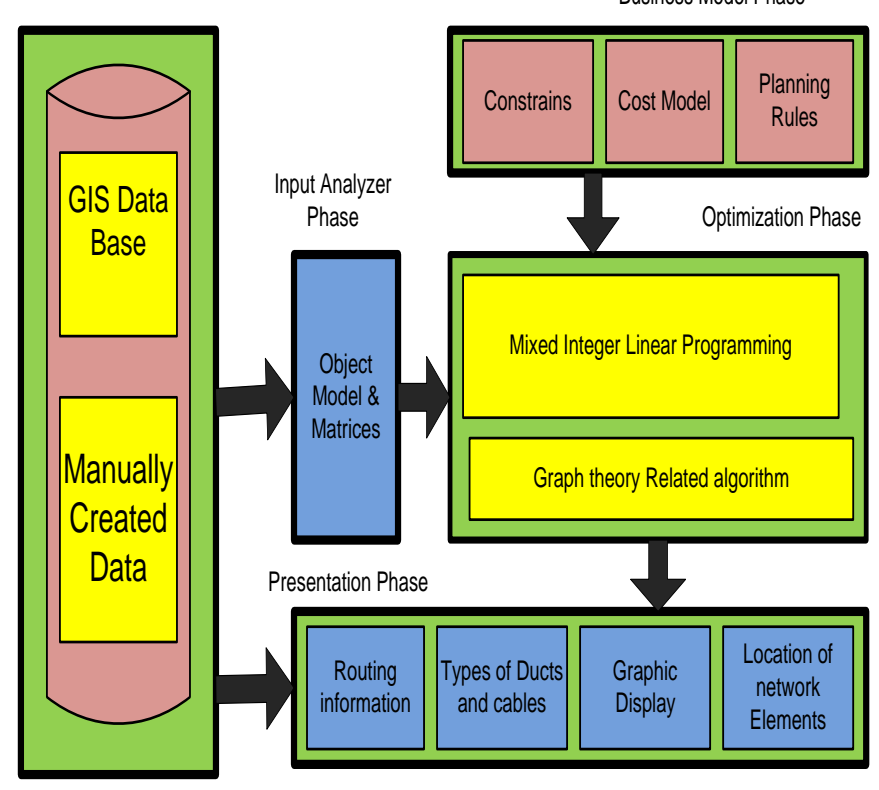

Fig. 4. Automation based Planning Process.
This Phase is especially problem particular as well as frequently modified to meet necessity. Moreover, the verification of this phase can be obtained by manual solution. Calculation of Complete expenditure and design limitation will validate in this phase. The business designer can validate their business model with the proposed Model. After the completion of business model, it can be tested on various optimization methods.

d) Network Optimization Phase: This phase consists of optimization approach based on Mixed Integer Linear Programming (MILP). It is a distinct variation of the linear programming. In MILP few variables have integer values. In MILP, our problem is molded by binary variables $[8,11,15]$.

Prior to executing the MILP-based design tool, we assumed that below given information's are delivered:

- Locations of customer sites.

- Location of one exchange $\mathrm{E}$.

- Possible sites of cable distribution (CD).

- Number of occupancies for all premises that determines number of required PON links.

- Civil layer network which specifies connectivity in different network components.

- Requirements of spare capacity needed to accommodate future network growth.

\section{Motivation}

In this paper, a network design tool for the GPON/FTTH network is proposed to automate the planning process. Thus, given the locations of customer plots, possible locations of CDs and SPs, the tool decides the optimal or near optimal locations of CDs and SPs taking into account the future growth and spare capacity distribution. In addition, cables are assigned from each plot to a CD and to the selected SP. The solution is optimal in the sense that it leads to the minimum cost of deployment which includes the number of network elements required, the total cabling distance and the installation costs.

By using an automated planning assistant tool, the planner can:

- Minimize network capital expenditure, i.e., installation and materials costs.

- Quickly achieve the network design of a given area.

- Compare what-if scenarios to meet changes in planning requirements.

- Rapidly re-cost networks for contract control and installation.

- Produce cable design and bill-of-materials automatically.

- Specify pre-determined locations for network elements prior to performing the network optimization. 
The benefits of automating, network design includes reducing installation and material expenses, decreasing time to make a design from hours to minutes, speedily re-costing networks for different laboring or equipment costs and making network design as well as automatically making bill-ofmaterials too.

\section{LITERATURE REVIEW}

\section{A. Literature Regarding FTTH Planning}

The issue of FTTH network planning has been identified in literature. In this section two key methodologies are frequently used. The first one is that meta-heuristic techniques such as genetic algorithms (GA) and exact techniques like use of Mixed Integer Linear programming (MILP) both methodologies have their pros and cons. The MILP can obtain global optimum solution as well as can outclass metaheuristics for a medium-sized network [5].

According to the authors of [6] presented a solution which employs heuristics, falling in $2^{\text {nd }}$ type of methodologies. Their research emphasized on networks that are multistage splitting. As they have complex problems, the researchers adopted heuristics for reducing computational time. The cluster based and random locations of splitters were taken and performed comparisons. The effect of different localization of splitters on lessening in CAPEX was deliberated. In [7] the authors proposed a model of a real life network. Real data of building sites and streets was mined from a Geographical Information System (GIS) by use of an open source map known as Open Street Map (OSM). This model was adapted to single level passive optical network (PON). These remote nodes are located for serving the customers. For more decrease costs sustained, cables channels shared by different routes of cable.

In [8], the networks that based on MILP, a design tool for GPON/FTTH networks are proposed. This tool automates the planning process of networks. By providing positions of customers and probable positions of SPs and DC's, it adopts optimum positions of DCs and SPs as well as assignment of cables in network elements and customers. Thus minimizes entire network development cost. The researchers took in to consideration future progression and introduced a technique for planning large networks.

According to [5], an approach is presented that is based on meta-heuristic. This approach used Ant Colony Optimization (ACO). This algorithm achieves cable assignment on a multilevel network which emphasizing on cost minimizing. This algorithm allocates customers to DCs as well as DCs to splitters simultaneously. Authors of [9] proposed a mixture of heuristics and mathematical programming for minimizing deployment cost of a GPON. The methodology of this research work was same to Simulated Annealing by which assignment of cables as well as positions of splitters recursively reallocated till a well cost was found. In [10], a scheme is presented that is based on previous class of methodologies. This scheme is a cross layer optimization. According to this scheme the researchers targets Greenfield network deployment and pursued to produce physical architecture of Wave Length Division Multiplexing (WDM) PON networks. It attains nominal cost for network deployment. This algorithm initially discovers optimum number of clusters for customers and then continues to assign every ONU to a cluster.

Researchers of [10] prolonged their research work in [11] by producing multiple WDM PON networks concurrently. It is attained by searching finest cost effective WDM PON by splitting an area in to sub sections in which every sub region covered by a specific PON. The authors of [12] proposed a tool for semi-automated network planning. It defines a suboptimal route distribution for deployment cost. It utilizes existing cable channels. After clustering customers, the authors of this research utilize GA for route deployment process. The outcomes are compared to network designs attained by manual process which depicted that in most cases this tool generates an inexpensive network.

Though, none of research work considered different types of network elements selection, such as research work in paper [8] emphasized on decisive optimum locations of network elements and cable assigning. They assumed a specific type of network element. Another heuristic approach was recently introduced in [13]. The approach is based on clustering and a Tabu search and has been enhanced with mechanisms handling resiliency issues as presented in [14]. The approach we use in this paper, originally presented in [15], is based on beam search [16]. This approach has been enhanced with mechanisms handling uncertainty issues following those used in [17] and upgraded with the MIP polishing mechanisms of [18]. This last idea to mix MIP methodology with heuristics proved to be very efficient and also has been recently used in [19]. The methodology used in [20, 21] is MIP facilitated by the use of valid inequalities and various algorithmic enhancements. Another recent work by Orange Labs is [22]. It is similar to our research in majority of assumptions and the methodology used. However, it covers only the last access part of FTTH network; thus, the authors do not consider splitting and OLT costs. Still, the detailed view on the fiber splicing problem presented in [22] is definitely worth noting.

\section{B. Literature Regarding GPON Technology}

There are two main streams of research focusing on GPON technology: Dynamic bandwidth allocation (DBA) algorithms among OLT and ONUs, which can be found in [23, 24, 25] and optimal network design of the physical layer for GPON deployment. The latter is the one considered in this research and discussed in detail. Using the classical operational research approach, the planning problems can be assumed of as a ordered concentrator network problems. In context of GPONs, the concentrator acts as a splitter to connect several ONUs to an OLT in a star topology. When several splitters are connected to the OLT at different locations, it becomes a double-star topology. Details of the classical access network design approach can be found in [26, 27, 28, 29].

In [30], the authors developed an optimization solution to perform multi-hierarchy PON planning. In their case, upper Optical Branching Devices (OBDs) and lower OBDs were introduced. The upper OBDs were used to connect between OLT and lower OBDs whilst lower OBDs were used to connect between ONUs and upper OBDs. 
The locations of OBDs were calculated based on the MaxMin Distance Cluster (MMDC) algorithm which can be found in [10]. Regarding the optimization framework, authors in [31] introduced a segmental framework which primarily emphasized on metaheuristic optimizing approaches. Practical sample from motorized domain comprised for validate how overall problem could be break down in to sub-jobs and controlled through propose framework.

According to [32], the author works on collective deployment of access network architectures such as Fiber To The Node (FTTN), Fiber To The Micro Node (FTTN) and Fiber To The Premise (FTTP) to decrease span of loops of coppers through use of DSL access multiplexer in external cabinet and field micro node which are nearer to subscribers. Several classes of services and subscribers per class per point of demand are considered. The MILP model has been proposed together with a tabular search base process for improving computational time needed for finding best resolution.

\section{Automation LeAding to Optimization}

MIP approach is usually using in different means in FTTH networks designing. According to our research work, we adopt MIP for the improvement of results that are returned by empirical algorithms of optimization framework that are introduced in [33]. Framework used: locations of demand, the available setup, with labor and equipment as well as technology restraints. It returns a complete network planning comprising: the topology of network, OLT, splices, splitters, OLT cards, cables, splices closures as well. By using stated aspects in modeling of a problem would leads to an incredible of variables and restraints making acquired model unsolvable by overall up-to-date MIP solvers. Hence, issue to be shortened for making it amenable. Key supposition was for using this approach is for improved acquired solutions; simplifying of model not be depends on neglecting ostensibly least significant factors, such as like splicing. While apparently additional significant factors, such as the OLT sites, to be static and detached from model. In this research, optimization of capital expenses essential for the placement of FTTH-OAN is addressed which contains one or more OLTs at the $\mathrm{CO}$ location and group of point of access that are located in the / or nearby to the CP locations. Networks gratify loads of all point of access take in to account permissible power budget of optical links and split scenarios.

\section{A. Prerequisites Data}

The problem that is denoted by $\Delta$ needs the given below input data:

- Passive and actives Equipment's record.

- Infrastructure networks Topology.

- Every distribution and access node infrastructure paths that are selected.

- All access nodes Signal demand.

- The Infrastructure sites that are decided for the installing active, passive equipment.

\section{B. Decision Variables}

For optimization, the decision variables are followed:

- Cabinet types utilization (given nodes)

- All types of Splitter with locations utilization

- All types of cables utilization (given topology)

- Splice closure and Splicing locations utilization

- OLT types splice closures (locations are given)

\section{Problem Statement}

Keeping in mind the structure as well as complexities of complete problem, in our research description of its formulation is split in to four (4) problems that are depicted in Fig. 5 as a square. We focused; these partial problems interlinked. The semantics of each variable that link to specific couples of partial problem is drawn by the shape of oval.

First part of problem is the bundle layer dimensioning denoted by $\Delta^{B l}$. Its purpose is that at assessing number with all types of splitters that are installed and at selection number of OLT cards to be install at Central Office (CO) nodes as well. Delivered resolution promises that all accessing nodes, irrespective of its distance to the $\mathrm{CO}$, delivered with requisite optical signal of appropriate power.

The $2^{\text {nd }}$ part of problem is cables which is denoted by $\Delta_{c a b}^{I}$ . It defines number of cables of all types that are installed at every infrastructure segment. Installed the cables are to provide fibers in numbers sustaining requests of bundle layer dimension problem.

Splices are the $3^{\text {rd }}$ part of problem. It is denoted by $\Delta_{S p l}^{I}$ which calculates number of optical closures and splices of all types which are essentially be install in every infrastructure node for supporting of solution that assessed in first two problems.

The fourth part of problem is site dimensioning. It is denoted by $\Delta_{\text {Sit }}^{I}$ that's goals is selecting a site type and number with all types of hardware's cabinet that are install in each infrastructure location.

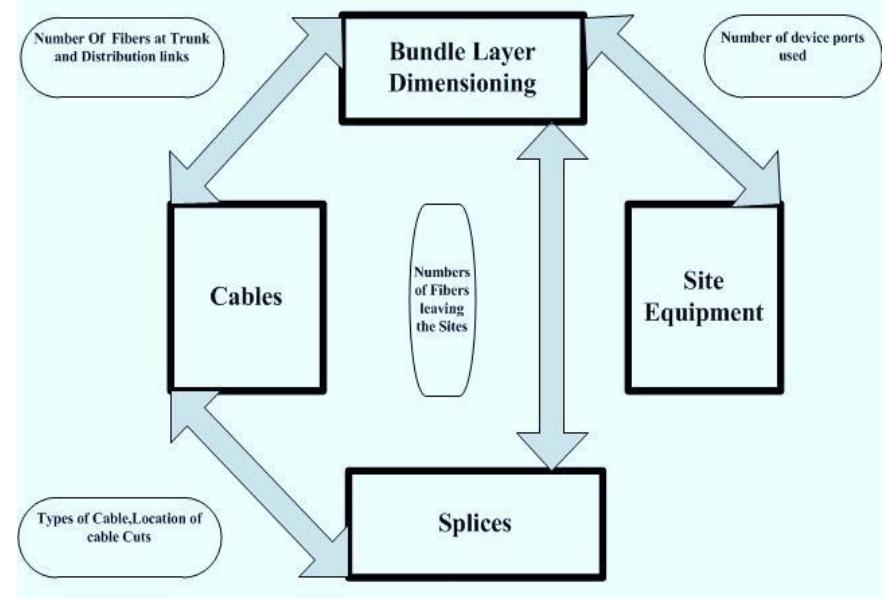

Fig. 5. Block Diagram of Proposed Structure for OLT. 


\section{Problem Objectives}

Key objective of $\mathcal{P}$ problem is to decrease the total cost is expressed by all partial of $\mathcal{P}$ :

$\Delta=\Delta^{B l}+\Delta_{c a b}^{I}+\Delta_{S p l}^{I}+\Delta_{S i t}^{I}$

In equation (1) $\partial$ depicting overall cost. The symbols $\Delta^{B l}$, $\Delta_{c a b}^{I}, \Delta_{S p l}^{I}$, and $\Delta_{\text {Sit }}^{I}$ are depicting cost of individual parts of $\mathcal{P}$ that are, bundle layer, cables, splices and site dimensioning respectively each problem.

\section{E. Description Layout of Problem}

To simplifying the overall layout, we are split problem in to two portions. The first part is the model part that is described in Section 6.1 which presents architectures with important notions that expresses overall organization of FTTH-OAN. The second part is equipment catalogue that is described in Section 6.2. This part describes a catalogue sets which identifies all equipment types. For every type, a set of critical parameters such as capacity, cost.

\section{MODEL OF FTTH- OAN}

This section presents a comprehensive model of all resources of FTTH-OANs. The basic FTTH-OAN model shown is in Fig. 6. It is appropriate for the requisites of formulation of the optimization problem. This model shown in Fig. 7 by dividing it into two fragments which are:

- Network

- Equipment

The network Fragment consist of equipment's permissible for installation in infrastructure nodes and segments such as cables, segments preparation types, hardware cabinets, OLT devices and cards, cable closures, splitters, etc.

\section{A. Network Fragments}

According to telecommunication modeling rules, like [34], we splitting networks fragment in to stack of layers. In these layers every pair neighboring layers, upper layer which is client, gains the benefits of all resource providing by the lowering layer which is server. We differentiate the four (4) layers starting from signal, bundle, infrastructure and cable layer.

Descriptions start from signal layering model. It contains an essential base for our optimizing methodology. It classifies components of signal nodes and links that are needed for delivering signal networks links in ONT and OLT devices. Unluckily, signaling model differentiates each individual component. Its direct application in formulation of optimizing problem leads to unsatisfactorily great optimization problem instances. To overwhelm this issue, we presented a layer of aggregated bundle model. It takes collection of signal nodes and connections as a bundle instead of distinct ones. As there is intricate relation in layers hence we settled their descriptions in an order which is not follows layers' order.

1) Model of infrastructure layer: The infrastructure layer model is illustrated in Fig. 8. This layer characterizes network of channels that are connected through staves which can accommodate optical cables that supports multiple ODNs. Network is needs to be linked therefore at least one link in Central Office and all ONT must be exist node.

Topologies of the infrastructure layers are molded by an undirected graph $G^{I L}=\left(\mathcal{N}^{I L}, \mathcal{L}^{I L}\right)$ with group of infrastructure nodes denoted by $\mathcal{N}^{I L}$ and group of undirected infrastructure link denoted by $\mathcal{L}^{I L} \subseteq \mathcal{N}^{I L} \times \mathcal{N}^{I L}$. Infrastructure node that is denoted by $n$ which is belongs to $\mathcal{N}^{I L}$, signifies a position, like a staves or a pole, in which optical wires sacked. The infrastructure link that is denoted by $l \in \mathcal{L}^{I L}$ signifies a place-holder, such as channel in pair of infrastructure node. All links can be accommodating different optical wires.

The set $\mathcal{N}^{I L S} \subseteq \mathcal{N}^{I L}$ of infrastructure sites is differentiated in such a way that nodes furnished to hold either signal splitters or active devices. These locations, depends on theirs positions in service area of networks, are apportioned in to central office sites $\mathcal{N}^{I L S O} \equiv\left\{n^{\text {ilso }}\right\}$, distribution point sites $\mathcal{N}^{I L S D}$ which is specify by $D P$, point of access locations $\mathcal{N}^{I L S A}$ that is indicated by $A P$ while the customer premise sites $\mathcal{N}^{I L S P}$ is depicted through $C P$.

Finally, a set $\mathcal{P}^{I L} \subseteq \mathcal{L}^{I L}$ is described that is the all infrastructure links with a suitable subset $\mathcal{P}^{I L S} \subset \mathcal{P}^{I L}$ of infrastructure trails; through supposition, for a couple.

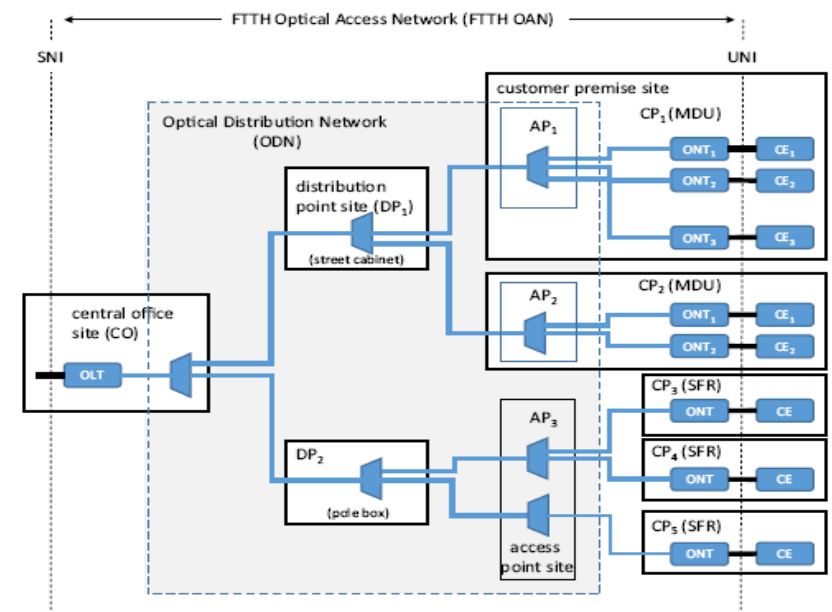

Fig. 6. FTTH -OAN Basic Network [33.]

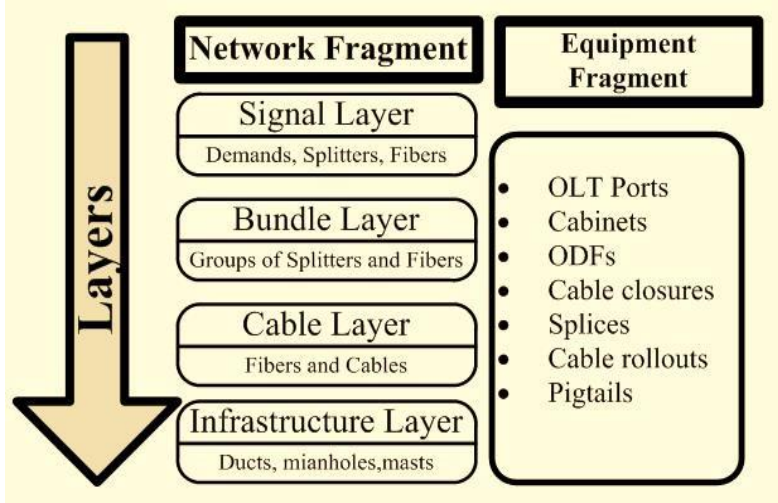

Fig. 7. Model Fragments [34]. 


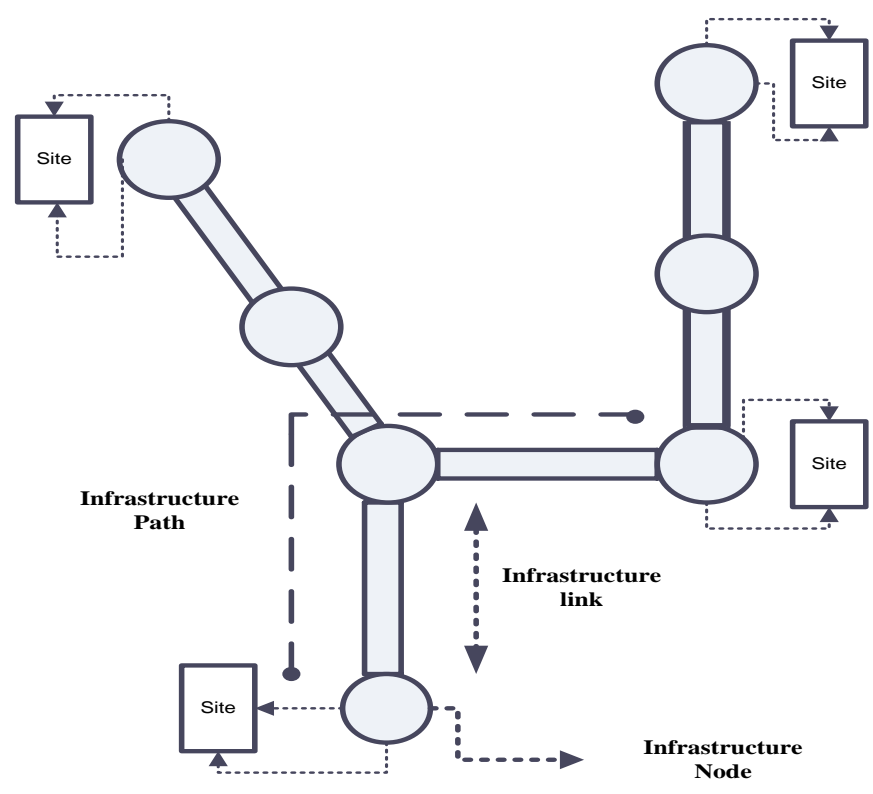

Fig. 8. Infrastructure Layer Topology.

$n_{1}, n_{2} \in \mathcal{N}^{I L S} \times \mathcal{N}^{I L S}$ of infrastructure locations. There is selected maximum one infrastructure link that denoted by $p \in$ $\mathcal{P}^{I L}: p \in \mathcal{P}^{I L S}$ denoted to as infrastructure trail of this pair.

2) Model of cable layer: Fig. 9 shows the fiber and cable layer Model. The Every fiber link contains a series of directed segments of fiber that are linked permanently or temporarily at the infrastructure node as optical splices or ODFs.

Lastly, optical cables segments are incessant segment of optical cables that is installed in infrastructures route containing single or multiple infrastructure segments.

3) Model of signal layer: Signaling layer gives a group of descendent signal network links in OLT devices ports, ONT terminals and group of parallel ascendant network links which links ONTs to OLTs for a single FTTH-OAN. These links are providing through the passive ODNs containing a set of signal splitters connected by dual directional fiber optic routes. The Signal nodes with links model is shown in Fig. 10. Signal layer topology is signified by a graph. This graph is denoted by $G^{S L}$ which is equal to signal node and group of directed signal connections.

Group of signal nodes that are denoted by $\mathcal{N}^{S L}$ and group of directed signal connections denoted by $\mathcal{L}^{S L}$. The signal nodes denote signal transporting functions are done through active as well as passive devices. The signal connections signify in turn distinct fiber optic routes interrelating couples of signal nodes. The $\mathcal{N}^{S L}$ is divided in to set head end nodes that is denoted by $\mathcal{N}^{S L H} \equiv\left\{n^{\text {slh }}\right\}$, set of access nodes denoted by $\mathcal{N}^{S L A}$, and set of signal distribution points denoted by $\mathcal{N}^{S L D}$. The set $\mathcal{N}^{S L D}$ is also divided into sets of distribution, head end and access signal distribution points that are denoted by $\mathcal{N}^{S L D H}, \mathcal{N}^{S L D D}$ and $\mathcal{N}^{S L D A}$ repectively. This division reveals level that engaged by specific splitters within network links. The $\mathcal{N}^{S L A}$ are real source of demanding signal demand of specific access node $n$ which belongs to $\mathcal{N}^{S L A}$. The $h^{\text {sl }}(n)$ is the number of network links that requires.

A signal node $\mathrm{n}$ belongs to $\mathcal{N}^{S L}$ depends on its class either $\mathcal{N}^{S L H}, \mathcal{N}^{S L D H}, \mathcal{N}^{S L D D}, \mathcal{N}^{S L D A}$, or $\mathcal{N}^{S L A}$ is defined a subset of infrastructure location types either $\mathcal{N}^{I L S O}, \mathcal{N}^{I L S D}, \mathcal{N}^{I L S A}$, or $\mathcal{N}^{I L S P}$ it can be installed in. For viable allocations, we used a function si_site(n) $: \mathcal{N}^{S L} \rightarrow \mathcal{N}^{I L S L}$, that for signal node $\mathrm{n} \in$ $\mathcal{N}^{S L}$, defines its presenting infrastructure location sl $\in \mathcal{N}^{I L S}$. By assumption following assignments are viable:

Single head end signal node $n^{\text {slh }}$ that belongs to $\mathcal{N}^{S L H}$ essentially be allotted to single $\mathrm{CO}$ site $n^{\text {ilso }}$ that belongs to $\mathcal{N}^{I L S O}$, where si_site $\left(n^{\text {slh }}\right) \equiv n^{\text {ilso }}$,

Every distribution signal distribution pointn belongs to $\mathcal{N}^{S L D D}$, can be allotted to CO site $n^{\text {ilso }}$ or any DP site sthat belongs to $\mathcal{N}^{I L S D}$ that is $\forall_{n \in} \mathcal{N}^{S L D D}$, si_site $(n) \in\left\{n^{i l s o}\right\} \cup \mathcal{N}^{I L S D}$,

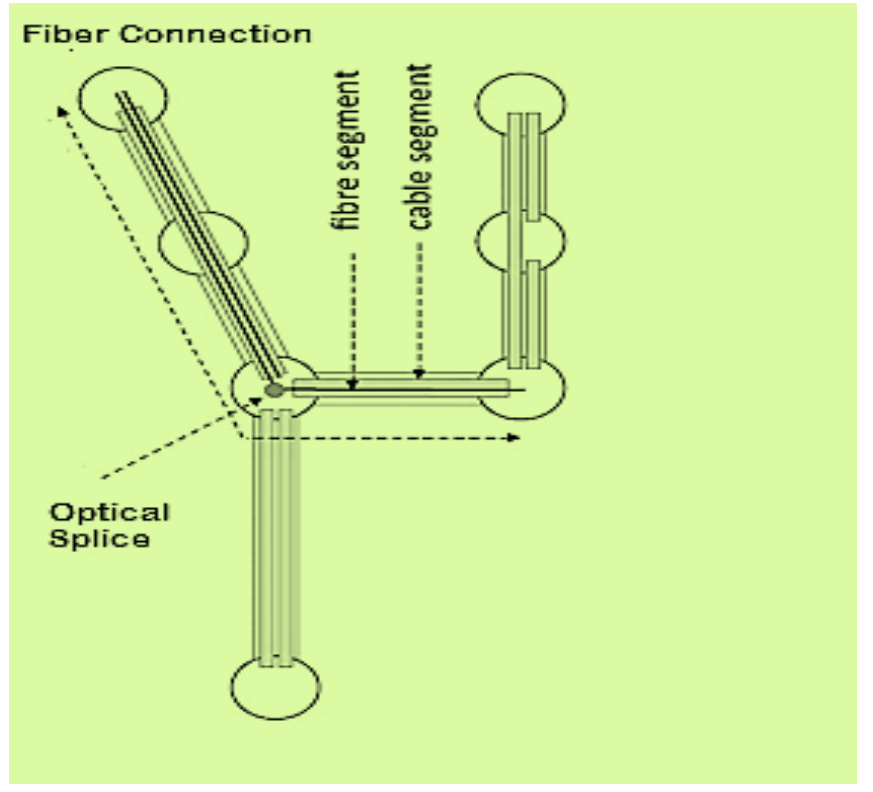

Fig. 9. Fibers and Cable Segment.

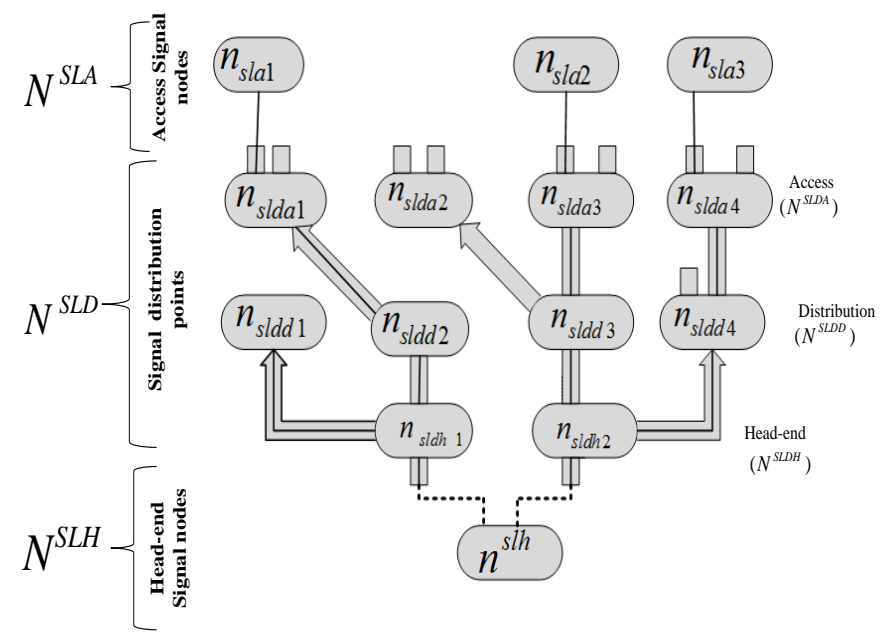

Fig. 10. Signal Nodes with Links and Connections. 
Each head end signal distribution point $n$ belongs to $\mathcal{N}^{S L D H}$ essentially be allotted to single CO site $n^{\text {ilso }}$, that is $\forall_{n \in} \mathcal{N}^{S L D H}$, si_site $(n) \equiv n^{\text {ilso }}$,

Every access signal distribution point $n$ that is belongs to $\mathcal{N}^{S L D A}$ can be allotted to any type of an infrastructure site however $\mathrm{CP}$, that is, si_site $(n) \in \mathcal{N}^{I L S O} \mathrm{U}$ $\mathcal{N}^{I L S D} \cup \mathcal{N}^{I L S A}$

Lastly, every access signal node $n$ that belongs to $\mathcal{N}^{S L A}$ essentially be allotted to any of CP infrastructure locations $s$ that is belongs to $\mathcal{N}^{I L S P}$

4) Model of bundle layer: The model of signaling layer recognizes each distinct component must deliver signal network links in OLT and ONT. In our work we presented aggregate model, by the name of bundle layer. It deliberates groups of signal nodes and links as a substitute of individual ones.

This model contains directed graph that is denoted by $G^{B L}$ which is equal to $\mathcal{N}^{B L}, \mathcal{L}^{B L}$. The bundle nodes denoted by $\mathcal{N}^{B L}$ while $\mathcal{L}^{B L}$ is group of bundle connections. $G^{B L}$ Organizes a contraction of a graph.

$G^{S L}=\left(\mathcal{N}^{S L}, \mathcal{L}^{S L}\right)$ of signaling layer, each bundle node $n_{b l}$ $\in \mathcal{N}^{B l}$ signifies subset $\mathcal{N}_{b l}^{s l} \subseteq \mathcal{N}^{s l}$ of signaling nodes. All bundle layer links $l_{b l} \in \mathcal{L}^{B L}: l_{b l} \in \delta\left(n_{b l}\right)$ occurrence of bundle node $n_{b l}$ signifies in turn group.

$\mathcal{L}_{b l}^{s l}=\left\{l_{s} \in \mathcal{L}^{s l}: l_{s l} \in \delta\left(\mathcal{N}_{b l}^{s l}\right)\right\}$ of each signaling link occurrence to this selected subset of signaling nodes. For simplify mapping in signaling and bundle layers model, we present functions $b s \_n m a p\left(n_{b l}\right): \mathcal{N}^{B L} \mapsto 2\left|\mathcal{N}^{S L}\right|$. This function expresses subset of signaling nodes $\mathcal{N}^{S L}$ amassed to bundles node $n_{b l} \in \mathcal{N}^{B L}$ and function $b s_{-} \operatorname{lmap}\left(\ln _{b}: \mathcal{L}^{B L} \mapsto\right.$ $2\left|\mathcal{L}^{S L}\right|$ which states subset of signaling links denoted by a bundle link $l_{b}$.

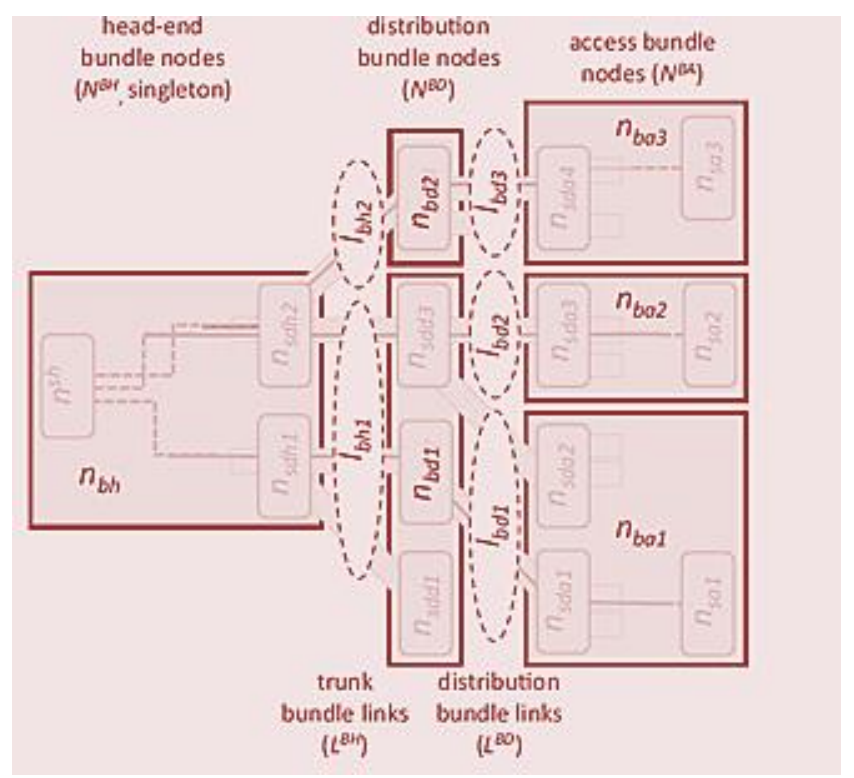

Fig. 11. Bundle Nodes and Bundle Links.
Following is categorization of bundle nodes:

a) Singleton set that represent by $\mathcal{N}^{B L H} \equiv\left\{n^{b l h}\right\}$ which masses the head end signal node $n^{\text {slh }}$. Each head end signaling distribution point $n_{\text {sldh }} \in \mathcal{N}^{S L D H}$, which is placed in $\mathrm{CO}$ infrastructure location $n^{\text {ilso }}$. Mentioning to sample network single bundle head-node $n^{\text {blh }}$ masses head end signal node $n^{\text {slh }}$ and two head end signal distribution points $n_{\text {sldh1 }}$ and $n_{\text {sldh2 }}$.

b) Set $\mathcal{N}^{B L D}$ of distribution bundle nodes. Every distribution bundle noden $n_{\text {bld }} \in \mathcal{N}^{B L D}$, agrees to CO or a DP infrastructure location $n_{i l s} \in \mathcal{N}^{I L S O} \cup \mathcal{N}^{I L S D}$ and groups each distribution signal distribution points $n_{\text {sldd }} \in \mathcal{N}^{S L D D}$ : si_site $\left(n_{\text {sldd }}\right)=n_{i l s}$, positioned in that location. In sample network, there are two distribution bundle nodes $n_{\text {bld1 } 1}, n_{\text {bld } 2}$ that collective, respectively, a subset of distribution signaling distribution points $\left\{n_{\text {sldd } 1}, n_{\text {sldd } 2}, n s n_{\text {sldd } 3}\right\}$ and $\left\{n_{\text {sldd } 4}\right\}$;set $\mathcal{N}^{B L A}$ of accessing bundle nodes. Every accessing bundle node $n_{b l a} \in \mathcal{N}^{B L A}$, related to an infrastructure location $n_{i l s} \in$ $\mathcal{N}^{I L S}$, and aggregates every access signal distribution point $n_{\text {slda }} \in \mathcal{N}^{S L D A}: \operatorname{si}$ _site $\left(n_{\text {slda }}\right)=n_{\text {ils }}$ as well as each accessing signaling node $n_{\text {sla }} \in \mathcal{N}^{S L A}:$ si_site $\left(n_{\text {sla }}\right)=n_{\text {ils }}$ nis positioned at this location. In sample network as shown in Fig. 11, in which three access bundle nodes represented by $n_{\text {bla1 } 1}, n_{\text {bla2 }}$, $n_{\text {bla3 }}$ respectively. We proposed $c(n): \mathcal{N}^{B L D} \mapsto 2\left|\mathcal{N}^{B L A}\right|$ function that for every distribution bundle node $n \in \mathcal{N}^{B L D}$ distinguish subset $\left\{m \in \mathcal{N}^{B L A}: \exists l \in \mathcal{L}^{B L D}, b \_a(l)=n \wedge b \_b(l)\right.$ $=m$ \} of bundle accessing nodes linked to that distribution node through a bundle connection; referred by distribution cone of $\mathrm{n}$ distributing nodes.

c) Bundle links that denoted by $\mathcal{L}^{B L}$ further divided into trunk bundle and distribution bundle links that are represented by $\mathcal{L}^{B L H}$ and $\mathcal{L}^{B L D}$ respectively. With support of bb_a(l) : $\mathcal{L}^{B L} \mapsto \mathcal{N}^{B L}$ and bb_b(l) : $\mathcal{L}^{B L} \mapsto \mathcal{N}^{B L}$ functions that recognize, correspondingly, start and end bundle nodes of a directed bundle link denoted by $1 \in \mathcal{L}^{B L}$, these two can be properly define as, $\mathcal{L}^{B L H}=\left\{1 \in \mathcal{L}^{B L}: \mathrm{bb} \_\mathrm{a}(\mathrm{l}) \in \mathcal{N}^{B L H}\right.$, bb_b(l) $\left.\in \mathcal{N}^{B L D}\right\}$ and $\mathcal{L}^{B L D}=\left\{1 \in \mathcal{L}^{B L}:\right.$ bb_a(l) $\in \mathcal{N}^{B L D}$, bb_b(l) $\in$ $\left.\mathcal{N}^{B L A}\right\}$ respectively, Sets $\mathcal{L}^{B L H}$ and $\mathcal{L}^{B L D}$ organize the segregating of set $\mathcal{L}^{B L}$.

The actual demands for signals network connections are generates in accessing bundle nodes $\mathcal{N}^{B L A}$; demand $h_{n}^{B}$ of every distinct accessing bundle node $n \in \mathcal{N}^{B L A}$ calculated by given below expression:

$h^{B}(n)=\sum_{s \in \mathcal{N}} S L A_{: s \in b s_{-} n \operatorname{map}(n)} h^{S}(s), \quad n \in \mathcal{N}^{B L A}$

5) Concluding remarks of network fragment: The direct bundle link $l$ that is belongs to $\mathcal{L}^{B L}$, of bundle layer maintained through a group of similar fibers links in si_site $\left(b b \_a(l)\right)$ and si_si te $\left(b b \_b(l)\right)$ infrastructure locations. By supposition, each fiber link used identical infrastructure trail $b i \_p(l) \in \mathcal{P}^{I L S}$ where function $b i \_p(l): \mathcal{L}^{B L} \mapsto \mathcal{P}^{I L S}$ expresses an infrastructure trail that is taken by each fiber link supportive bundle link. 
Let directed trunk bundle connection from group $\mathcal{L}^{\mathrm{BLH}}$ Each fiber link which supports that link is known as trunk fiber link. It uses a trunk infrastructure trail which contains trunk fiber segments. All cable segments that have trunk fibers denoted as Trunk cable segment. Conferring to stated rules, we introduced group of trunk infrastructures trails representing by $\mathcal{P}^{\mathrm{ILSH}}$ and group of distribution infrastructure trails that represented by $\mathcal{P}^{\mathrm{ILSD}}$. We signify group of trunk infrastructure segment and distribution infrastructure segment through, respectively, $\mathcal{L}^{I L H} \subseteq \mathcal{L}^{I L}$ and $\mathcal{L}^{I L D} \subseteq \mathcal{L}^{I L}$; we focused on sets $\mathcal{L}^{I L H}$ and $\mathcal{L}^{I L D}$ that normally don't organize segregating of set $\mathcal{L}^{I L}$. We assumed that each trunk infrastructure trail from $\mathcal{P}^{I L S H}$ which use single trunk infrastructure segment from $\mathcal{L}^{I L H}$ traverses segment in identical direction. Therefore, trunk and distribution, infrastructure segment can be considered as directed. $i i_{-} a(l)$ : $\mathcal{L}^{I L} \mapsto \mathcal{N}^{I L}$ and $i i \_b(l): \mathcal{L}^{I L} \mapsto \mathcal{N}^{I L}$, functions expresses respectively, start and ending infrastructure nodes of infrastructure segments. The trunk segment in $\mathcal{L}^{I L H}$ creates a directed tree by root at $\mathrm{CO}$ location $n^{\text {ilso }}$, whereas distribution segments in set $\mathcal{L}^{I L D}$ creates forest of directed tree, that all rooted at a location which hosts distribution bundle node from $\mathcal{N}^{B L D}$ set. Hence, for every trunk segment $l$ that belongs to $\mathcal{L}^{I L H}$ there is just one or none predecessor trunk segment ii_ah $(l)$. Likewise, for every distribution segment $k$ that belongs to $\mathcal{L}^{I L D}$ there is just one or none predecessor distribution segment $i i_{-} a d(k)$.

\section{B. Equipment Catalogue Portion}

In this section with the help of catalog defining the physical equipment types are acceptable for installation at infrastructure nodes and sites. Every catalog set is denoted by $\delta$ with a lowercase upper index; it is also used for individual properties of an example of a particular type. Parameters common to every type, like cost or capacity, are denoted by Greek letters $\delta$ and $\eta$ with appropriate upper indices. A brief list of catalogue sets is listed in Table IV. The detail description of this fragment is beyond the scope of this paper.

TABLE. IV. EQUiPMENT CATAlogue Sets

\begin{tabular}{|l|l|}
\hline Name & Catalogue \\
\hline$\delta^{o a}$ & Optical cables \\
\hline$\delta^{o b}$ & OLT cards \\
\hline$\delta^{o c}$ & Cabinets \\
\hline$\delta^{o d}$ & Sites \\
\hline$\delta^{o e}$ & Segment preparations \\
\hline$\delta^{o f}$ & OLT devices \\
\hline$\delta^{o g}$ & Fiber Splices \\
\hline$\delta^{o h}$ & Optical Splitter \\
\hline$\delta^{o i}$ & Splitter Combinations \\
\hline$\delta^{o j}$ & Joint Closures \\
\hline &
\end{tabular}

\section{CONCLUSION AND FUTURE WORK}

The increasing demand for Broad band internet services requires adaption of novel fiber base technologies. To attract new customers, fixed access network operators have to substantially increase the speed and quality of internet services. This can only be achieved by bringing the fiber as close to the customer as possible. This requires extensive planning in term of cost, time and infrastructure. In this paper we have presented a model of automating FTTH planning considering OAN. Different features, planning phases and model fragments have been identified and discussed, both theoretically as well as mathematically. In our future work we will present formulation in term of optimization for FTTH cost effective deployment. Real world experiment will aid in formulation as well as validation.

\section{REFERENCES}

[1] J. Prat, Next-generation FTTH passive optical networks: research towards unlimited bandwidth access. Springer, 2008.[Online] Available: http://dx.doi.org/10.1007/978-1-4020-8470-6.

[2] PR Newswire,http://www.prnewswire.com/news-releases-62266647 .html,"April 2011.

[3] M.M.Al-Quzwini,-Design and Implementation of a Fiber To The Home FTTH Access Network based on GPON, in International Journal of Computer Applications, vol.92,no.6, April 2014.

[4] ITU-T G.984 Gigabit Passive Optical Network Specifications

[5] A. Chu, K. F. Poon, and A. Ouali, "Using Ant Colony Optimization to design GPON-FTTH networks with aggregating equipment," in 2013 IEEE Symposium on Computational Intelligence for Communication Systems and Networks (CIComms), April 2013, pp. 10-17.

[6] A. Eira, J. Pedro, and J. Pires, "Optimized Design of Multistage Passive Optical Networks," IEEE/OSA Journal of Optical Communications and Networking, vol. 4, no. 5, pp.

[7] O. Kipouridis, C. Machuca, A. Autenrieth, and K. Grobe, "Street aware infrastructure planning tool for Next Generation Optical Access networks," in 2012 16th International Conference on Optical Network Design and Modeling (ONDM), April 2012, pp. 1-6.

[8] K. F. Poon and A. Ouali, "A MILP based design tool for FTTH access networks with consideration of demand growth," in 2011 International Conference for Internet Technology and Secured Transactions (ICITST), Dec 2011, pp. 544-549.

[9] J. Li and G. Shen, "Cost Minimization Planning for Greenfield Passive Optical Networks," IEEE/OSA Journal of Optical Communications and Networking, vol. 1, no. 1, pp. 17-29, June 2009.

[10] R. Chowdhury and B. Jaumard, "A cross layer optimization scheme for WDM PON network design and dimensioning," in 2012 IEEE International Conference on Communications (ICC), June 2012, pp. $3110-3115$.

[11] "A p-center optimization scheme for the design and dimensioning of a set of WDM PONs," in 2012 IEEE Global Communications Conference (GLOBECOM), Dec 2012, pp. 2977-2983.

[12] B. Lakic and M. Hajduczenia, "On optimized Passive Optical Network (PON) deployment," in Second International Conference on Access Networks Workshops, 2007. Access Nets '07, Aug 2007, pp. 1-8.

[13] G. V. Arévalo, R. C. Hincapié, and R. Gaudino, "Optimization of multiple PON deployment costs and comparison between GPON, XGPON, NGPON2 and UDWDM PON," Opt. Switching Netw., vol. 25, no. Supplement C, pp. 80-90, 2017. 
[14] G. V. Arévalo and R. Gaudino, "A techno-economic network planning tool for PON deployment including protection strategies," in 19th Int. Conf. on Transparent Optical Networks (ICTON), July 2017, pp. 1-4.

[15] R. Bisiani, "Beam search," in Encyclopedia of Artificial Intelligence, Wiley, 1987, pp. 56-58.

[16] M. Żotkiewicz and M. Mycek, "Impact of demand uncertainty models on FTTH network design," in 18th Int. Conf. on Transparent Optical Networks (ICTON), July 2016, pp. 1-4.

[17] Mycek M, Pióro M, Żotkiewicz M. MIP model for efficient dimensioning of real-world FTTH trees. Telecommunication Systems. 2018 Jun 1: PP 1-20.

[18] F. D’Andreagiovanni, F. Mett, A. Nardin, and J. Pulaj, "Integrating LPguided variable fixing with MIP heuristics in the robust design of hybrid wired-wireless FTTx access networks," Appl. Soft Comput., vol. 61, no. Supplement C, pp. 1074-1087, 2017.

[19] Fritzsche, Lutz, Mathias Schweigel, and Rong Zhao. "Integrated Network Planning: A Key Success Factor for Network Operators." In Future Telco, pp. 43-52. Springer, Cham, 2019.

[20] Hervet, C., Faye, A., Costa, M.C., Chardy, M., \& Francfort, S. (2013). Solving the two-stage robust FTTH network design problem under demand uncertainty. In Proceedings of the international network optimization conference. Costa Adeje, Spain.

[21] Angilella, Vincent, Matthieu Chardy, and Walid Ben-Ameur. "Fiber cable network design in tree networks." European Journal of Operational Research 269, no. 3 (2018): 1086-1106.

[22] Angilella, V. (2018). Optimal design of Fiber To The Home networks (Doctoral dissertation, Institut National des Télécommunications)

[23] C. Bock, P. Chanclou, J. Finochietto, G. Franzl, M. Hajduczenia, T. Koonen, P. Monteiro, F. Neri, J. Prat, and H. Silva, "Artchitecture of future access networks," in Next-Generation FTTH Passive Optical Networks: Research towards unlimited bandwidth access, 2008, pp. 546.
[24] B. Skubic, J. Chen, J. Ahmed, L. Wosinska, and B. Mukherjee, "A comparison of dynamic bandwidth allocation for EPON, GPON, and next-generation TDM PON," IEEE Journals, vol. 47, pp. 40-48, 2009.

[25] B. Chen, J. Chen, and S. He, "Efficient and fine scheduling algorithm for bandwidth allocation in ethernet passive optical networks," IEEE J. Sel. Topics Quantum Elect., vol. 12, no. 4, pp. 653 -660, 2006.

[26] C. Lee, "An algorithm for the design of multi-type concentrator networks," J. Oper. Res. Soc., vol. 44, pp. 471-482, 1993.

[27] P. McGregor and D. Shen, "An algorithm for the access facility location problem," IEEE Trans. Commun., vol. 25, pp. 61-73, 1977.

[28] H. Pirkul and V. Nagarajan, "Locating concentrators in centralized computer networks," Annals of Operations Research, vol. 36, pp. 61-73, May 1992.

[29] S. Narasimhan and H. Pirkul, "Hierarchial concentrator location problem," Computer Communications, vol. 15, pp. 185-191, March 1992.

[30] M. Lv and X. Chen, "Heuristic based multi-hierarchy passive optical network planning"in Wireless Communications, Networking and Mobile Computing, 2009.WiCom '09. 5th International Conference on, sept. 2009, pp. 1-4.

[31] M. Lukasiewycz, M. Glaß, F. Reimann, and J. Teich, "Opt4J - A Modular Framework for Meta-heuristic Optimization," in Proceedings of the Genetic and Evolutionary Computing Conference (GECCO 2011), Dublin, Ireland, 2011.

[32] C. Steve, "Designing low cost access networks with iptv performance constraints," in Next Generation Internet Networks, 2008. NGI 2008, 2008 , pp. $45-52$.

[33] Zotkiewicz, M., Mycek,M., \& Tomaszewski, A. (2016). Profitable areas in large-scale FTTH network optimization. Telecommunication Systems, 61(3), 591-608.

[34] ITU-T. (2001). Generic Functional Architecture of Transport Networks. Tech. rep. Recommendation G.805. 